\title{
Zu den Autorinnen
}

Katajun Amirpur wurde 1971 als Tochter eines iranischen Vaters und einer deutschen Mutter in Köln geboren. Sie studierte Islamwissenschaften und Politologie in Bonn und Teheran und promovierte über den Islamtheoretiker Abdolkarim Soroush. Seit Mai 2003 ist sie Stipendiatin des Emmy Noether-Programms der DFG und forscht über zeitgenössische schiitische Koranexegese. Andere Forschungsschwerpunkte: Zivilgesellschaft, Reformtheologie, Medien.

Rose Marie Beck, 1964, Dr. phil., wissenschaftliche Mitarbeiterin an der Universität Frankfurt. Afrikanistin mit den Schwerpunkten Swahili, Populäre Kultur, Kommunikationswissenschaft, Soziolinguistik, Oralistik. Habilitationsprojekt über „sprechende Objekte“ im afrikanischen Vergleich. Aktuell beschäftigt in einem Projekt (VW-Stiftung) zu ,Language, Gender, and Sustainability“.

Priska Furrer, geboren 1957, Studium der Neueren vorderorientalischen Philologie, Islamwissenschaft und Neueren Geschichte an der Universität Bern, Dissertation und Habilitationsschrift im Bereich der modernen türkischen Literatur, langjährige Tätigkeit in der interkulturellen Erziehung, seit 2003 Privatdozentin für türkische Sprache, Geschichte und Kultur an der Universität Bern. Zahlreiche Publikationen zur modernen türkischen Literatur.

Iris Hermann, Studium der allgemeinen Literaturwissenschaft, Geschichte und Germanistik in Bonn, Recife (Brasilien) und Bielefeld. Arbeitet z. Zt. als Hochschulassistentin an der Univ. Bielefeld. Publikationen zu Kleist, Jean Paul, Trakl,
Kafka, Lasker-Schüler, Wittgenstein, zur Psychoanalyse und zur Literaturtheorie.

Dietlind Hüchtker, Dr. phil, Historikerin, wissenschaftliche Mitarbeiterin am Geisteswissenschaftlichen Zentrum Geschichte und Kultur Ostmitteleuropas, Leipzig. Forschungsschwerpunkte: Frauen- und Geschlechtergeschichte, Feministische Theorie und Cultural Studies. Arbeitet an einem Projekt zu Geschlecht und Macht in den Politiken politischer Bewegungen in Galizien.

Alexandra Karentzos, Dr. des.; wissenschaftliche Assistentin an den staatlichen Museen zu Berlin, lehrt Kunstwissenschaft an der HBK Braunschweig. Ihre Arbeitsschwerpunkte liegen in der Kunst des 19. bis 21. Jahrhunderts und den Gender Studies. Sie hat die Ausstellung Parastou Forouhar: Tausendundein Tag in der Nationalgalerie im Hamburger Bahnhof - Museum für Gegenwart, Berlin mitkuratiert.

Christa Kersting, Dr. phil., Priv.-Doz. für Erziehungswissenschaft an der Freien Universität Berlin, Vertretungs- bzw. Gastprofessur an den Universitäten Bielefeld und Passau. Forschungsschwerpunkte: Wissenschaftsgeschichte, -politik und Disziplingeschichte der Pädagogik (insb. 18. und 20. Jahrhundert, u. a. zur Remigration nach 1945); Ideen- und Kulturgeschichte; Frauen- und Geschlechterforschung.

Almut Kirschbaum, geb. 1973. DiplomSozialwissenschaftlerin und wiss. Mitarbeiterin in einem Forschungsprojekt zum Thema »Promotionsförderung und Geschlecht" an der Carl von Ossietzky-Uni- 
versität Oldenburg; Arbeitsschwerpunkte: Gleichstellung im Wissenschaftssystem, Geschlechterforschung, Organisationssoziologie, empirische Sozialforschung.

Carmen Klement, Dipl.-Soz., studierte Soziologie/Rechtswissenschaft an den Universitäten Bremen und Helsinki. Derzeit Mitarbeiterin im Fachbereich Sozialwissenschaften der Universität der Bundeswehr in München. Arbeitsschwerpunkte: Zukunft der Arbeit, Geschlechterforschung, Arbeitsmarkttheorien, Sozialpolitik und Methoden des internationalen Vergleichs.

Renate Kreile, Dr. rer. soc. habil., geb. 1950. Studium der Germanistik und Ev. Theologie in Tübingen und Berlin. Seit 1977 Unterrichtstätigkeit an verschiedenen Gymnasien in Baden-Württemberg. Zweitstudium der Politikwissenschaft und Soziologie in Tübingen. 1991 Promotion. Seit 1996 Privatdozentin am Institut für Politikwissenschaft der Universität Tübingen. Zahlreiche Veröffentlichungen zu politischer Herrschaft und Geschlechterpolitik im Vorderen Orient.

Ulrike Lingen-Ali studierte DiplomPädagogik an der Carl von OssietzkyUniversität Oldenburg sowie Arabisch an der Universität Birzeit, Palästina. Beruflich war sie im Bereich der stationären und ambulanten feministischen Sozialarbeit tätig. Zur Zeit arbeitet sie an ihrer Dissertation über "Weiblichkeitsentwürfe in Palästina und in Deutschland aus soziokultureller und komparativer Perspektive«.

Ann Elizabeth Mayer, PhD, JD, Associate Professor of Legal Studies an der Wharton School der Universität von Pennsylvania, USA. Sie ist Expertin für Recht in den Gesellschaften des Nahen Ostens, für islamisches Recht und Menschenrechte. Ann
E. Mayer war für das Lawyers Committee on Human Rights als Beraterin in Menschenrechtsfragen im Nahen Osten tätig und gehört zahlreichen Gremien an, die sich mit Menschenrechten und Middle Eastern Studies beschäftigen.

Mona Motakef hat Sozialwissenschaften und Interkulturelle Pädagogik in Oldenburg studiert. Sie schreibt ihre Diplomarbeit über eine Bildungskooperation im Kontext von Post-Apartheid und PostKolonialismus und arbeitet im Projekt "Geschlechterkonstruktionen und Gewalt« sowie als freie Journalistin bei der tageszeitung Bremen.

Susanne Rauscher, geb. 1973, Musikliebhaberin und PR-Redakteurin. Studierte Politikwissenschaft, Volkswirtschaftslehre, Soziologie und Sozialpsychologie in Frankfurt am Main. Lebt und arbeitet in Hamburg.

Silke Reuter, M.A., Studium der Romanistik und Soziologie an den Universitäten Bremen und Paris; wissenschaftliche Mitarbeiterin im Zentrum für Sozialpolitik der Universität Bremen, Schwerpunkt "Strukturwandel von Erwerbsarbeit und familialen Lebensformen und Sozialstaatsreform in Frankreich".

Angelika Schaser, Dr. phil., Professorin für Neuere Geschichte an der Universität Hamburg. Forschungs- und Veröffentlichungsschwerpunkte: Sozialgeschichte der Frühen Neuzeit, Liberalismusforschung, Geschichte der deutschen Frauenbewegung, Nation und Geschlecht.

Tanja Scheiterbauer studierte Islamwissenschaft und Politikwissenschaft an den Universitäten Bonn und Marburg. Sie promoviert über die islamistische Frauenbewegung in der Türkei im Rahmen des Graduiertenkollegs Öffentlichkeiten und 
Geschlechterverhältnisse. Dimensionen von Erfahrung an der Goethe-Universität Frankfurt a. $M$.

Stevie Schmiedel, Medien- und Kulturwissenschaftlerin, promovierte im März 2003 an der Postgraduate School of Critical Theory and Cultural Studies der Universität Nottingham mit einer Arbeit über feministische Kulturkritik und Gilles Deleuze.

Gesine Spieß, promovierte Erziehungswissenschaftlerin, seit 1994 Professorin an der Fachhochschule Erfurt mit den Schwerpunkten Kindheit, Sozialisation und $\mathrm{Ge}$ schlechterverhältnis. Veröffentlichungen u.a. zur politischen Bildung von Frauen in den neuen Bundesländern, zu Frauen in Führungspositionen, zu Frauenarmut und zu Gender in Lehre und Didaktik.
Anna Maria Stuby, Dr. phil., lehrt englische Literaturwissenschaft an der Universität Hannover. Schwerpunkte: Symbol- und Mythenforschung, Biografieforschung, Moderne.

Anja Weiß, Dr. phil., geb. 1968, Sozialwissenschaftlerin an der Universität der Bundeswehr München, Leiterin des DFGProjekts "Hochqualifizierte Migrant/-innen. Zur Transnationalisierung sozialer Lagen«. Arbeitsschwerpunkte: Theorien sozialer Ungleichheit; Rassismus; interkulturelle Konflikte und Migration.

Siegrid Wieczorek, Sozialwissenschaftlerin, wissenschaftliche Mitarbeiterin im DFGProjekt „Flexible Arbeitszeit- und Teilzeitmodelle" am Institut für Politikwissenschaft der Justus-Liebig-Universität Gießen. Arbeitsschwerpunkte: Internationale Frauenpolitik, Internationale Arbeitsteilung, Globalisierung, Empirische Sozialforschung. 